\title{
Localized versus extended systems in density functional theory: Some lessons from the Kohn-Sham exact exchange potential
}

\author{
C. M. Horowitz, ${ }^{1}$ C. R. Proetto, ${ }^{2,3, *}$ and J. M. Pitarke ${ }^{4,5}$ \\ ${ }^{1}$ Instituto de Investigaciones Fisicoquímicas Teóricas y Aplicadas, (INIFTA), CCT La Plata-CONICET, UNLP, Sucursal 4, \\ Casilla de Correo 16, La Plata, Argentina \\ ${ }^{2}$ Institut für Theoretische Physik, Freie Universität Berlin, Arnimallee 14, D-14195 Berlin, Germany \\ ${ }^{3}$ European Theoretical Spectroscopy Facility (ETSF) \\ ${ }^{4}$ CIC NanoGUNE Consolider, Tolosa Hiribidea 76, Donostia, E-20018 Basque Country, Spain \\ ${ }^{5}$ Materia Kondenstsatuaren Fisika Saila, Donostia International Physics Center, Centro Física Materiales (CSIC)-UPV/EHU, \\ 644 Posta Kutxatila, Bilbo, E-48080 Basque Country, Spain
}

(Received 12 January 2010; revised manuscript received 3 March 2010; published 26 March 2010)

\begin{abstract}
A long-standing puzzle in density functional theory is the issue of the long-range behavior of the KohnSham exchange-correlation potential at metal surfaces. As an important step toward its solution, it is proven here, through a rigorous asymptotic analysis and an accurate numerical solution of the optimized-effectivepotential integral equation, that the Kohn-Sham exact exchange potential decays as $\ln (z) / z$ far into the vacuum side of an extended semi-infinite jellium. In contrast with the situation in localized systems, such as atoms, molecules, and slabs, this dominant contribution does not arise from the so-called Slater potential. This exact exchange result provides a strong constraint on the suitability of approximate correlation-energy functionals.
\end{abstract}

DOI: 10.1103/PhysRevB.81.121106

PACS number(s): 71.15.Mb, 31.15.eg, 71.10.Ca

\section{INTRODUCTION}

In their seminal density-functional-theory (DFT) investigation of the electronic structure of metal surfaces, Lang and Kohn ${ }^{1}$ pointed out that far outside the surface the KohnSham (KS) exchange-correlation $(x c)$ potential $V_{x c}(z)$ of DFT should behave like the classical image potential $-e^{2} / 4 z$, with $z$ being the distance from the surface. While from the physical viewpoint this suggestion is attractive and reasonable, 40 years later its rigorous proof is still an open question. Two kinds of approaches are possible to address this difficult problem. One, followed already by some authors, ${ }^{2-4}$ is to consider exchange and correlation contributions to the KS $x c$ potential together; we note, however, that as the correlation contribution should always be approximated, great care must be taken in approximating the corresponding exchange contribution in a compatible way. Within this context, it is not surprising that various asymptotics have been suggested for the KS exchange-only ( $x$-only) potential along this pathway. ${ }^{2-4}$

A second way to proceed, followed in the present Rapid Communication, is to exploit the fact that since the exchange-energy functional is known exactly, the corresponding KS exchange potential $V_{x}(z)$ can also be known exactly, by using the optimized-effective-potential (OEP) method of DFT. ${ }^{5}$ Moreover, knowing the exact $V_{x}(z)$, the analysis of the more elusive $\mathrm{KS}$ correlation potential $V_{c}(z)$ may be advanced on firmer grounds than previously. We have succeeded along this second type of approach by proving rigorously that the asymptotic behavior of the KS exchange potential far into the vacuum side of a semi-infinite (SI) jellium is of the form $\ln (z) / z$. This analytical result is supported by a fully self-consistent numerical solution of the OEP integral equation, which describes accurately the KS exact exchange potential at the bulk, interface, and vacuum regions of our semi-infinite system.

\section{BASIC OEP EQUATIONS FOR A METAL SURFACE}

The calculations presented below focus on the SI jellium model of a metal surface, where the discrete character of the positive ions inside the metal is replaced by a uniform distribution of positive charge (the jellium). The positive jellium density is given by $n_{+}(z)=\bar{n} \theta(-z)$, which describes a sharp jellium $(z<0)$ vacuum $(z>0)$ interface at $z=0$. The model is invariant under translations in the $x, y$ plane (of normalization area $A$ ), so the single-particle KS orbitals of DFT can be rigorously factorized as $\varphi_{\mathbf{k}_{\|}, k}(\mathbf{r})=e^{i \mathbf{k}_{\|} \cdot \boldsymbol{\rho}} \xi_{k}(z) / \sqrt{A L}$, where $\boldsymbol{\rho}$ and $\mathbf{k}_{\|}$are the in-plane coordinate and wave vector, respectively. $k$ and $L$ refer to the remaining (continuous) quantum number and the normalization length, both along the $z$ direction. The effective one-dimensional KS spin-degenerate orbitals $\xi_{k}(z)$ are the solutions of the KS differential equation (atomic units are used throughout)

$$
\hat{h}_{\mathrm{KS}}^{k}(z) \xi_{k}(z):=\left[-\frac{1}{2} \frac{\partial^{2}}{\partial z^{2}}+V_{\mathrm{KS}}(z)-\varepsilon_{k}\right] \xi_{k}(z)=0,
$$

where $\varepsilon_{k}$ are the KS eigenvalues, and $V_{\mathrm{KS}}(z)=V_{\mathrm{H}}(z)+V_{x c}(z)$. $V_{\mathrm{H}}(z)$ is the Hartree potential, and $V_{x c}(z):=\delta E_{x c} / \delta n(z)$, with $E_{x c}:=E_{x c}\left[\left\{\varepsilon_{k}\right\},\left\{\xi_{k}\right\}\right]$ and $V_{x c}(z)$ being the $x c$ energy functional and potential, respectively, and $n(z)$ as the ground-state electron density. The OEP integral equation whose solution provides the KS $x c$ potential $V_{x c}(z)$ is compactly given as ${ }^{6}$

$$
\int_{0}^{k_{F}}\left(k_{F}^{2}-k^{2}\right) \Psi_{k}^{*}(z) \xi_{k}(z) d k+\text { c.c. }=0 .
$$

Here, $\Psi_{k}(z)$ are the so-called orbital shifts, defined by

$$
\Psi_{k}(z)=\int_{-\infty}^{\infty} \xi_{k}\left(z^{\prime}\right) \Delta V_{x c}^{k}\left(z^{\prime}\right) G_{k}\left(z^{\prime}, z\right) d z^{\prime},
$$

with 


$$
G_{k}\left(z, z^{\prime}\right)=\frac{1}{\pi} P \int_{0}^{k_{F}} \frac{\xi_{k^{\prime}}^{*}(z) \xi_{k^{\prime}}\left(z^{\prime}\right)}{\left(\varepsilon_{k}-\varepsilon_{k^{\prime}}\right)} d k^{\prime}
$$

being the KS Green's function, $\Delta V_{x c}^{k}(z)=V_{x c}(z)-u_{x c}^{k}(z)$, and $u_{x c}^{k}(z)=\left[4 \pi / A\left(k_{F}^{2}-k^{2}\right) \xi_{k}^{*}(z)\right] \delta E_{x c} / \delta \xi_{k}(z) ; u_{x c}^{k}(z)$ are usually referred to as orbital-dependent $x c$ potentials. The symbol " $P$ " in Eq. (4) denotes the "principal value" and $k_{F}$ represents the magnitude of the Fermi wave vector. ${ }^{7}$ The exact $V_{x c}(z)$ entering Eq. (2) is obtained as the solution of this integral equation, which must be solved self-consistently together with Eq. (1).

While for formal discussions the integral form of the OEP equation [Eq. (2)] is useful, it is often more convenient to recast it in the following fully equivalent form, after a wellestablished sequence of transformations: $:^{5,6}$

$$
V_{x c}(z)=V_{x c}^{\mathrm{KLI}}(z)+V_{x c}^{\mathrm{Shift}}(z),
$$

where $V_{x c}^{\mathrm{KLI}}(z)$ represents the so-called Krieger-Li-Iafrate (KLI) contribution, ${ }^{8,9}$

$$
V_{x c}^{\mathrm{KLI}}(z)=\int_{0}^{k_{F}} \frac{\left|\xi_{k}(z)\right|^{2}}{2 \pi^{2} n(z)}\left[u_{x c}^{k}(z)+\overline{\Delta V}_{x c}^{k}\right] \widetilde{d k},
$$

and

$$
V_{x c}^{\text {Shift }}(z)=-\int_{0}^{k_{F}} \frac{\left[\left(k_{F}^{2}-k^{2}\right) \Psi_{k}(z) \xi_{k}(z)+\Psi_{k}^{\prime}(z) \xi_{k}^{\prime}(z)\right]}{2 \pi^{2} n(z)} \widetilde{d k},
$$

with $\widetilde{d k}=\left(k_{F}^{2}-k^{2}\right) d k$, primes denoting derivatives with respect to the coordinate $z$ and the ground-state electron density $n(z)$ being given by the following expression:

$$
n(z)=\frac{1}{2 \pi^{2}} \int_{0}^{k_{F}}\left(k_{F}^{2}-k^{2}\right)\left|\xi_{k}(z)\right|^{2} d k .
$$

Now we focus on the exchange contribution $V_{x}(z)$ to the KS $x c$ potential of Eq. (5), as obtained by replacing the orbital-dependent $x c$ potentials $u_{x c}^{k}(z)$ entering Eqs. (6) and (7) by their $x$-only counterparts $u_{x}^{k}(z)$ which are known exactly. ${ }^{10}$ In this case, the first term on the rhs of Eq. (6) is easily recognized to be twice the position-dependent exchange energy per particle $\varepsilon_{x}(z)$, defined as the interaction between a given electron at $z$ and its exact exchange hole. ${ }^{10}$ Noting that $2 \varepsilon_{x}(z)=V_{x}^{\mathrm{S}}(z)$, with $V_{x}^{\mathrm{S}}(z)$ being the so-called Slater potential, ${ }^{8}$ we write

$$
V_{x}(z)=V_{x}^{\mathrm{S}}(z)+V_{x}^{\Delta}(z)+V_{x}^{\mathrm{Shift}}(z),
$$

where $V_{x}^{\Delta}(z)$ represents the contribution to the exchange potential $V_{x}(z)$ from the $x$-only counterpart of the second term on the rhs of Eq. (6).

\section{NUMERICAL RESULTS}

In the case of the SI jellium, we have achieved the selfconsistent numerical solution of the $x$-only counterparts of Eqs. (1) and (5). The KS equations have been solved by following the procedure explained in Ref. 10; the orbital

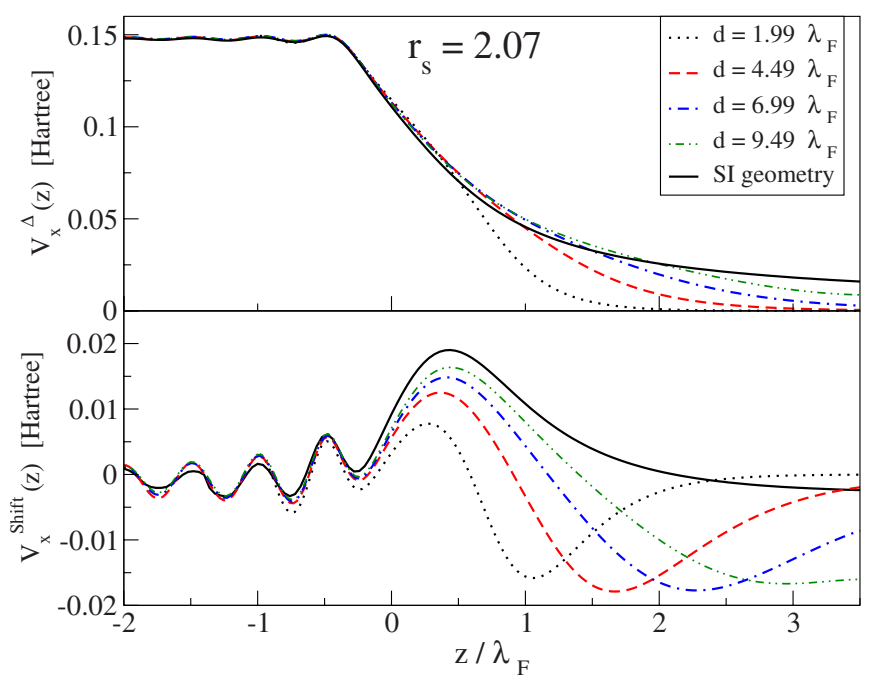

FIG. 1. (Color online) OEP self-consistent calculations of $V_{x}^{\Delta}(z)$ (top panel) and $V_{x}^{\text {Shift }}(z)$ (lower panel) for the SI geometry (solid line) and jellium slabs of various thicknesses $d$, for $r_{s}=2.07$. $\lambda_{F}=2 \pi / k_{F}$ is the Fermi wavelength. The jellium-vacuum interface is at $z=0$.

shifts were directly calculated from its definition in Eq. (3), with the KS Green's function computed using the procedure of Ref. 11 .

The correct asymptotics (at $z \rightarrow \infty$ ) of the Slater potential $V_{x}^{\mathrm{S}}(z)$ have been reported for a SI jellium ${ }^{12-14}$ and for jellium slabs, ${ }^{10}$ with the result that $V_{x}^{\mathrm{S}}(z)$ decays in both cases as $-\beta / z$ but with a coefficient $\beta$ that in the case of a SI jellium is electron-density-dependent while for jellium slabs $\beta=1$. Hence, here we focus on the remaining contributions: $V_{x}^{\Delta}(z)$ and $V_{x}^{\text {Shift }}(z)$.

OEP self-consistent calculations of $V_{x}^{\Delta}(z)$ and $V_{x}^{\text {Shift }}(z)$ for an electron-density parameter $r_{s}$ corresponding to the average density of valence electrons in $\mathrm{Al}\left(r_{s}=2.07\right)$ are plotted in Fig. 1 for the SI geometry and for jellium slabs of various thicknesses. We note that the bulk limit is correctly reproduced: $V_{x}^{\Delta}(z \rightarrow-\infty)=k_{F} / 2 \pi \simeq 0.148$ Hartree, ${ }^{15}$ while $V_{x}^{\text {Shift }}(z \rightarrow-\infty)$ presents small oscillations around zero, as it should be. In the case of jellium slabs, the numerical results were obtained using the procedure followed in Ref. 6.

In the vacuum, both $V_{x}^{\Delta}(z)$ and $V_{x}^{\text {Shift }}(z)$ decay exponentially for jellium slabs, as discussed before. ${ }^{6}$ In the case of a SI jellium, however, the decay of $V_{x}^{\Delta}(z)$ and $V_{x}^{\text {Shift }}(z)$ turns out to be qualitatively different. This is seen in Fig. 2, where these quantities are plotted for $r_{s}=6$, together with the Slater potential $V_{x}^{\mathrm{S}}(z)$ and the total $V_{x}(z)$. This figure shows that in the case of an extended SI jellium the asymptotics of $V_{x}(z)$ are dominated by $V_{x}^{\Delta}(z)$, which at large $z$ is positive.

\section{ANALYTICAL ASYMPTOTICS}

In order to determine the actual asymptotic behavior of $V_{x}(z)$ in the vacuum region of a SI jellium, we first appeal to the asymptotic analysis of the KS orbitals $\xi_{k}(z)$ entering Eq. (1). One finds ${ }^{13,14}$ 


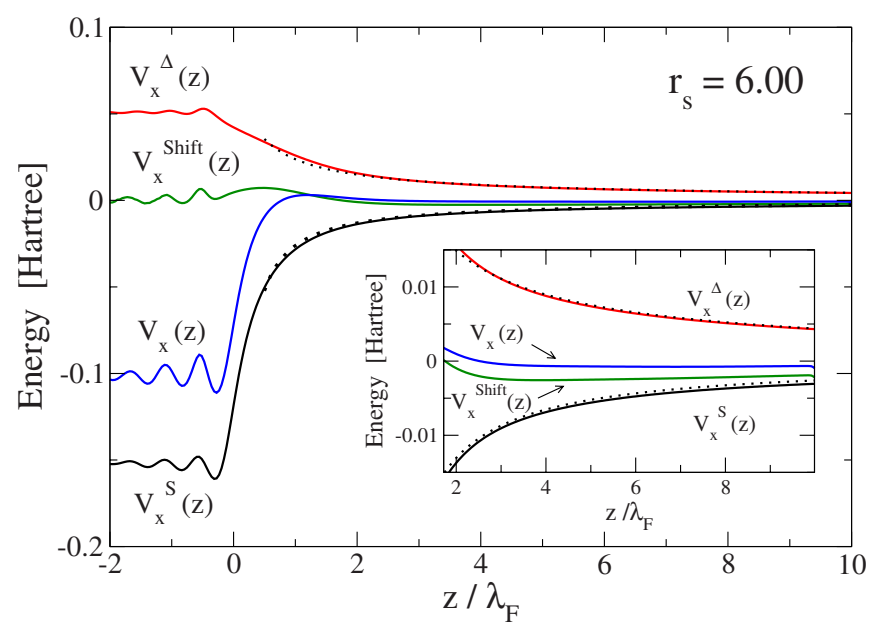

FIG. 2. (Color online) OEP self-consistent calculations of $V_{x}^{\mathrm{S}}(z), V_{x}^{\Delta}(z)$, and $V_{x}^{\mathrm{Shift}}(z)$ for the SI geometry and $r_{s}=6$. The jellium-vacuum interface is at $z=0$. The bulk limits for $V_{x}^{\mathrm{S}}(z), V_{x}^{\Delta}(z)$, and $V_{x}(z)$ are correctly reproduced: $V_{x}^{\mathrm{S}}(z \rightarrow-\infty)$ $=-3 k_{F} / 2 \pi \simeq-0.153$ Hartree (Ref. 16), $\quad V_{x}^{\Delta}(z \rightarrow-\infty)=k_{F} / 2 \pi$ $\simeq 0.051$ Hartree, and $V_{x}(z \rightarrow-\infty)=-k_{F} / \pi \simeq-0.102$ Hartree. The analytical asymptotes of Eqs. (12) and (14) are shown by dotted lines. An enlarged view of the far-vacuum region is exhibited in the inset.

$$
\xi_{k}(z \rightarrow \infty) \rightarrow \xi_{k_{F}}(z \rightarrow \infty) e^{-\alpha z \Delta k}
$$

and

$$
n(z \rightarrow \infty) \rightarrow \frac{3 \bar{n}}{4\left(\alpha k_{F} z\right)^{2}}\left|\xi_{k_{F}}(z \rightarrow \infty)\right|^{2},
$$

with $\alpha=k_{F} / \sqrt{2 W}$, with $W$ being the work function, and $\Delta k=k_{F}-k$. This result is perfectly plausible: for $z k_{F} \gg 1$, the only $k$ 's that matter are those close to $k_{F}$ and such that $z \Delta k \sim 1$, so the "window" for the relevant $k$ 's decreases linearly with distance. As for the electron density, it is interesting to note that it decays with an extra power $z^{2}$ in the denominator that is absent in the case of jellium slabs; ${ }^{10}$ this is due to the fact that as $z k_{F} \gg 1$ the factor $\left(k_{F}^{2}-k^{2}\right)$ in the integrand of Eq. (8) becomes effectively small, while in the case of jellium slabs this factor is always finite and the electrondensity decay is purely exponential. This anticipates that qualitative differences might be expected between the asymptotics of localized (slabs) and extended (SI) systems.

By introducing Eqs. (10) and (11) into the expression for the Slater potential [the $x$-only counterpart of the first term on the rhs of Eq. (6)], one finds the known result ${ }^{10,12-14}$

$$
V_{x}^{\mathrm{S}}(z \rightarrow \infty)=-\frac{(\pi+2 \alpha \ln \alpha)}{\pi\left(1+\alpha^{2}\right)} \frac{1}{z} .
$$

Solamatin and Sahni (SS) (Ref. 12) then derived the asymptotic structure of $V_{x}(z)$ from an approximate form of the so-called Sham-Schlüter integral equation relating $V_{x}(z)$ to the nonlocal Hartree-Fock self-energy and concluded that the asymptotics of $V_{x}(z)$ are embodied by half the Slater potential; i.e., $V_{x}(z \rightarrow \infty)=\frac{1}{2} V_{x}^{\mathrm{S}}(z \rightarrow \infty)$. SS supported their result by applying the definition of $V_{x}(z)$ as the functional de- rivative of the exchange energy (expressed in terms of the Slater potential),

$$
V_{x}(\mathbf{r})=\frac{1}{2} V_{x}^{\mathrm{S}}(\mathbf{r})+\frac{1}{2} \int d \mathbf{r}^{\prime} n\left(\mathbf{r}^{\prime}\right) \frac{\delta V_{x}^{\mathrm{S}}\left(\mathbf{r}^{\prime}\right)}{\delta n(\mathbf{r})},
$$

and then suggesting that the contribution of the second term of Eq. (13) in the vacuum region is zero in the leading order of $1 / z$. It is well known, however, that the second term of Eq. (13) contains another term of the form $\frac{1}{2} V_{x}^{\mathrm{S}}(\mathbf{r}),{ }^{15}$ leading, therefore, to an expression for $V_{x}(z)$ that contains the full Slater potential $V_{x}^{\mathrm{S}}(z)$ and not one-half of it, as noted by Nastos $^{13}$ and correctly given in Eq. (9) above. Moreover, here we prove that at large $z$ the full $V_{x}(z)$ of Eq. (9) is not dominated by the Slater potential $V_{x}^{\mathrm{S}}(z)$ but by $V_{x}^{\Delta}(z)$ instead.

Here we have succeeded, by introducing Eqs. (10) and (11) into the $x$-only counterpart of the second term on the rhs of Eq. (6), in obtaining the following neat (positive) expression for the leading contribution of $V_{x}^{\Delta}(z)$ to the long-range exchange potential:

$$
\begin{aligned}
V_{x}^{\Delta}(z \rightarrow \infty) & =\int_{0}^{k_{F}} \frac{\overline{\Delta V}_{x}^{k}}{2 \pi^{2} n(z \rightarrow \infty)} \mid \xi_{k}\left(\left.z \rightarrow \infty\right|^{2} \widetilde{d k}\right. \\
& =\frac{1}{2 \pi \alpha z}\left[\ln \left(\alpha k_{F} z\right)+C\right],
\end{aligned}
$$

where $C \sim 0.96351$. In passing from the first to the second line we have replaced $\Delta V_{x}^{k}(z)$, which enters the calculation of $\overline{\Delta V}_{x}^{k}$, by its bulk value. That is, $\Delta V_{x}^{k}(z) \simeq \Delta V_{x}^{k}(z \rightarrow-\infty)$ $=-k_{F} / \pi-u_{x}^{k}(z \rightarrow-\infty)$. The explicit (analytic) expression for $u_{x}^{k}(z \rightarrow-\infty)$ is obtained through a $\mathbf{k}_{\|}$Fourier transform of the orbital-dependent exchange potential of a three-dimensional electron gas. ${ }^{16}$

As for $V_{x}^{\text {Shift }}(z)$, we have first analyzed the asymptotics of Eq. (2) and then applied to it the operator $\hat{h}_{\mathrm{KS}}^{k_{F}}(z)$. Solving the resulting equation for $V_{x}(z \rightarrow \infty)$, one recovers the asymptotic expressions for $V_{x}^{S}(z)$ and $V_{x}^{\Delta}(z)$ given by Eqs. (12) and (14), and one also obtains

$$
\begin{aligned}
V_{x}^{\text {Shift }}(z \rightarrow \infty)= & \frac{\alpha^{2} z^{2}}{k_{F} \xi_{k_{F}}(z \rightarrow \infty)} \int_{0}^{k_{F}} \Delta k e^{-\alpha z \Delta k} \\
& \times\left[\left(k_{F}-\frac{\alpha^{2} \Delta k}{2}\right)+\alpha \frac{\partial}{\partial z}\right] \Psi_{k}(z \rightarrow \infty) \widetilde{d k}
\end{aligned}
$$

At this point, we need $\Psi_{k}(z \rightarrow \infty)$, which we obtain from the asymptotics of the orbital-shifts differential equation $\hat{h}_{\mathrm{KS}}^{k}(z) \Psi_{k}(z)=-\left[\Delta V_{x}^{k}(z)-\overline{\Delta V}_{x}^{k}\right] \xi_{k}(z)$. Noting that at $z k_{F} \gg 1$ all contributions in $\Delta V_{x}^{k}(z)$ tend to zero, the asymptotics of the orbital shifts are found to be given by the following expression:

$$
\Psi_{k}(z \rightarrow \infty) \rightarrow[f(k)+z g(k)] \xi_{k}(z \rightarrow \infty),
$$

where the first and second terms on the rhs are, respectively, the homogeneous and particular solutions of the orbital-shifts differential equation at $z \rightarrow \infty$. Here, $2 g(k)=-\overline{\Delta V}_{x}^{k} /(\sqrt{2 W}+\alpha \Delta k)$, and the explicit expression for $f(k)$ is not needed. After introduction of Eq. (16) into Eq. 
(15), we find that $V_{x}^{\text {Shift }}(z \rightarrow \infty)$ decays as $\ln (z) / z^{2}$. Hence, putting this together with Eqs. (12) and (14), we conclude that far outside a semi-infinite jellium the KS exact exchange potential decays as follows:

$$
V_{x}(z \rightarrow \infty)=\frac{\ln \left(\alpha k_{F} z\right)}{2 \pi \alpha z} .
$$

Equation (17) represents the main result of this work. The asymptotics of Eqs. (12) and (14) are plotted in Fig. 2 (dotted lines), showing that they are in excellent quantitative agreement with our fully self-consistent OEP numerical calculations at $z>\lambda_{F}$. In retrospect, the result of Eq. (17) looks natural for the SI case. For slabs, Eq. (9) yields $V_{x}(z \rightarrow \infty)=-1 / z+\overline{\Delta V}_{x}^{m}$, with $m$ being the quantum number corresponding to the highest occupied slab level; ${ }^{6}$ the first contribution is brought by the slab Slater potential $V_{x}^{S}(z)$, and the second contribution [brought by the slab $V_{x}^{\Delta}(z)$ ] is a constant which is chosen to be zero. ${ }^{5,6}$ In the SI case, however, while it is still true that the quantity $\overline{\Delta V}_{x}^{k}$ entering Eq. (14) is zero, the non-negligible contribution from $\overline{\Delta V}_{x}^{k}$ at $k_{F}-k<1 / z$ yields a $V_{x}^{\Delta}(z)$ potential that decays as $\ln (z) / z$ and dominates the asymptotics of the full $V_{x}(z)$.

\section{CONCLUSIONS}

In summary, we have solved a long-standing problem relative to the long-range behavior of the KS exact exchange potential at metal surfaces as an important step toward the understanding of the actual asymptotic behavior of the full KS $x c$ potential. Through a rigorous asymptotic analysis and an accurate numerical solution of the OEP integral equation, we have shown that far into the vacuum side of a semiinfinite jellium the KS exact exchange potential decays as $\ln (z) / z$ (positive). This analytical result, which does not arise from the Slater potential and is supported by a fully selfconsistent numerical solution of the OEP integral equation, is in contrast with the situation in localized systems, such as atoms, molecules, and slabs; as in the case of finite systems, for jellium slabs the asymptotics of the KS exchange potential arise from the full Slater potential, which decays as $-1 / z .{ }^{6}$ Finally, we note that due to the fact that the full KS $x c$ potential of a semi-infinite metal should be expected to be absent of the dominant $\ln (z) / z$ exchange asymptotics, our exact exchange result provides a strong constraint on the suitability of approximate correlation-energy functionals.

\section{ACKNOWLEDGMENTS}

C.M.H. acknowledges financial support from CONICET of Argentina. C.R.P. was supported by the EC's Marie Curie IIF (Grant No. MIF1-CT-2006-040222). J.M.P. acknowledges partial support by the University of the Basque Country, the Basque Unibertsitate eta Ikerketa Saila, and the Spanish Ministerio de Educación y Ciencia (Grants No. FIS2006-01343 and No. CSD2006-53).
*Permanent address: Centro Atómico Bariloche and Instituto Balseiro, 8400 S. C. de Bariloche, Río Negro, Argentina.

${ }^{1}$ N. D. Lang and W. Kohn, Phys. Rev. B 1, 4555 (1970).

${ }^{2}$ O. Gunnarsson, M. Jonson, and B. I. Lundqvist, Phys. Rev. B 20, 3136 (1979), on page 3154, it is argued that at points far outside a metal surface (large $z$ ) the KS potential decays faster than $-e^{2} / z$ when only exchange effects are included.

${ }^{3}$ C.-O. Almbladh and U. von Barth, Phys. Rev. B 31, 3231 (1985), on page 3236, it is argued that for macroscopic systems the exchange potential tends exponentially to zero, concluding that long-range components of $V_{x c}(z)$ can only originate from correlation effects.

${ }^{4}$ L. J. Sham, Phys. Rev. B 32, 3876 (1985), by analyzing the asymptotic limit of the so-called Sham-Schlüter integral equation for $V_{x c}(z)$, it is concluded that the exchange potential at points far outside a metal surface is of the form $\sim-e^{2} / z^{2}$, in disagreement with Ref. 3.

${ }^{5}$ T. Grabo, J. Kreibich, S. Kurth, and E. K. U. Gross, in Strong Coulomb Interactions in Electronic Structure Calculations: Beyond the Local Density Approximation, edited by V. I. Anisimov
(Gordon and Breach, Amsterdam, 2000).

${ }^{6}$ C. M. Horowitz, C. R. Proetto, and S. Rigamonti, Phys. Rev. Lett. 97, 026802 (2006); C. M. Horowitz, C. R. Proetto, and J. M. Pitarke, Phys. Rev. B 78, 085126 (2008).

${ }^{7} k_{F}=(9 \pi / 4)^{1 / 3} / r_{s}$ with $r_{s}$ being (in units of the Bohr radius) the radius of a sphere containing on average one electron: $r_{s}$ $=(3 / 4 \pi \bar{n})^{1 / 3}$.

${ }^{8}$ J. B. Krieger, Y. Li, and G. J. Iafrate, Phys. Rev. A 46, 5453 (1992).

${ }^{9}$ Mean values are defined as $\bar{O}^{i}=\int \xi_{k}^{*}(z) O^{i}(z) \xi_{k}(z) d z$. For simplicity, we choose $\xi_{k}^{*}(z)=\xi_{k}(z)$.

${ }^{10}$ C. M. Horowitz, L. A. Constantin, C. R. Proetto, and J. M. Pitarke, Phys. Rev. B 80, 235101 (2009).

${ }^{11}$ A. Liebsch, J. Phys. C 19, 5025 (1986).

${ }^{12}$ A. Solomatin and V. Sahni, Phys. Rev. B 56, 3655 (1997).

${ }^{13}$ F. Nastos, Ph.D. thesis, Queen's University, 2000.

${ }^{14}$ Z. Qian and V. Sahni, Int. J. Quantum Chem. 104, 929 (2005).

${ }^{15}$ See, e.g., O. Gritsenko, R. van Leeuwen, E. van Lenthe, and E. J. Baerends, Phys. Rev. A 51, 1944 (1995).

${ }^{16}$ J. Bardeen, Phys. Rev. 49, 653 (1936). 